\title{
Drug-induced thrombocytopenia associated with trastuzumab in a patient with HER2-positive recurrent gastric cancer
}

\author{
Yuko Takano $^{1}{ }^{10} \cdot$ Satoshi Furune ${ }^{1} \cdot$ Yuki Miyai $^{1} \cdot$ Sachi Morita $^{1} \cdot$ Megumi Inoue $^{1} \cdot$ Tomoya Shimokata $^{1}$. \\ Mihoko Sugishita ${ }^{1} \cdot$ Ayako Mitsuma $^{1} \cdot$ Osamu Maeda $^{1} \cdot$ Yuichi Ando $^{1}$
}

Received: 5 September 2021 / Accepted: 19 October 2021 / Published online: 2 November 2021

(c) The Author(s) 2021

\begin{abstract}
Here, we report a 57-year-old female patient with HER2-positive recurrent gastric cancer who experienced drug-induced thrombocytopenia associated with trastuzumab, a humanized anti-HER2 monoclonal antibody. Shortly after the initiation of S-1, oxaliplatin, and trastuzumab chemotherapy, the patient experienced severe thrombocytopenia and did not respond to platelet transfusions. Based on the findings of increased numbers of polynuclear megakaryocytes in the bone marrow and an elevated level of platelet-associated $\operatorname{IgG}(\mathrm{PA}-\operatorname{IgG})$, the patient was diagnosed with drug-induced thrombocytopenia (DITP). The platelet count recovered rapidly with oral prednisolone $(1 \mathrm{mg} / \mathrm{kg})$. Since we initially suspected oxaliplatin as the causal agent, S-1 was restarted as a monotherapy, followed by trastuzumab after a 3-week interval, without oxaliplatin. On the second day after the addition of trastuzumab, severe thrombocytopenia occurred again, which suggests that trastuzumab was responsible for the DITP. The patient no longer experienced severe thrombocytopenia during the subsequent S-1 and oxaliplatin chemotherapy, which supports this hypothesis.
\end{abstract}

Keywords Trastuzumab · Drug-induced thrombocytopenia $\cdot$ DITP $\cdot$ Gastric cancer

\section{Introduction}

The humanized anti-human epidermal growth factor 2 (HER2) monoclonal antibody trastuzumab is a key drug in first-line chemotherapy regimens for HER2-positive advanced gastric or gastro-esophageal junction cancers. Trastuzumab occasionally causes hypersensitivity reactions, also known as infusion reactions, and latent or overt cardiac dysfunction after long-term treatment, whereas this drug rarely causes hematological toxicity or myelosuppression, which are common among cytotoxic anticancer drugs. Here, we report a case of acute, severe thrombocytopenia that was likely associated with trastuzumab.

Yuko Takano

y.takano@med.nagoya-u.ac.jp

1 Department of Clinical Oncology and Chemotherapy, Nagoya University Hospital, Nagoya, Japan

\section{Case presentation}

A 57-year-old female patient was admitted to our hospital for swollen para-aortic lymph nodes detected by abdominal ultrasonography during a routine medical checkup. Thirteen years earlier, the patient had undergone total gastrectomy for T1bN1M0 Stage IB (AJCC 7th edition) gastric cancer. The patient did not receive adjuvant chemotherapy. She was also treated with entecavir for chronic hepatitis B and denosumab for osteoporosis and had received mecobalamin as an oral vitamin B12 supplement after total gastrectomy. $H$. pylori infection was not assessed. She also had no history of allergic reaction to medications or substances. A pathologic examination of CT-guided needle biopsy specimens from the para-aortic lymph nodes revealed HER2-positive moderately differentiated adenocarcinoma that was consistent with the metastatic lymph nodes from gastric cancer 13 years earlier (Fig. 1a). The patient was then diagnosed with HER2-positive recurrent gastric cancer.

The patient began treatment with first-line chemotherapy consisting of oral S-1 (a combination of tegafur, gimeracil, and oteracil potassium) at a dose of $50 \mathrm{mg}$ twice daily on a 2-week on and 1-week off schedule, intravenous oxaliplatin 

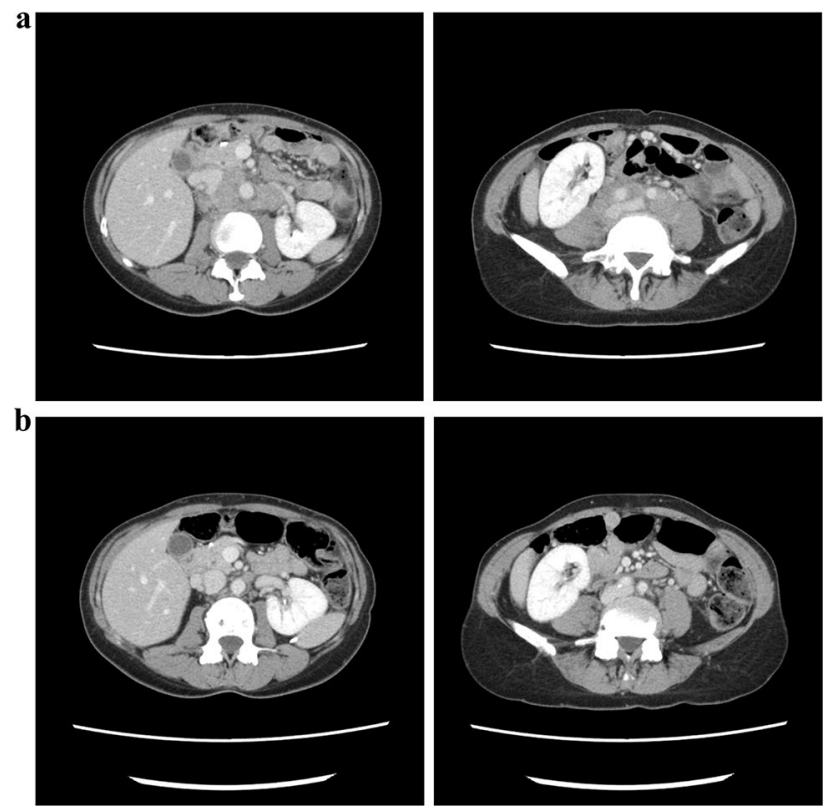

Fig. 1 Abdominal CT image prior to treatment (a) and after the second infusion of trastuzumab (b). The para-aortic lymphadenopathy shrunk remarkably after treatment $\left(100 \mathrm{mg} / \mathrm{m}^{2}\right)$, and trastuzumab $(8 \mathrm{mg} / \mathrm{kg}$ as a loading dose followed by $6 \mathrm{mg} / \mathrm{kg}$; CT-P6, Nippon Kayaku, Tokyo, Japan) given on day 1 of a 3-week cycle. Platelet count was $293,000 / \mu \mathrm{L}$, and the other blood chemistry and hematologic findings were within the normal range at the time of chemotherapy initiation. Oxaliplatin and trastuzumab infusions were successfully completed; however, a grade 1 infusion reaction occurred during trastuzumab infusion. The patient was discharged the following day (day 2). At a follow-up outpatient visit on day 8 , purpura and petechiae that appeared shortly after discharge were observed throughout the patient's body. She was also aware of macrohematuria that was persistent since day 3 . Hematologic tests revealed grade 4 thrombocytopenia, a platelet count of $1000 / \mu \mathrm{L}$, and moderate anemia with a hemoglobin level of $8.1 \mathrm{~g} / \mathrm{dL}$, while other blood chemistry and hematologic findings, such as white blood cell counts and neutrophil to lymphocyte ratio, were within normal range. According to the patient and her family, S-1 had been suspended since day 2 .

The patient was hospitalized immediately and received repeated platelet transfusions, but her platelet count did not recover (Fig. 2a). Based on the findings of increased
Fig. 2 Changes in platelet count following the first (a) and second (b) trastuzumab infusions. Prednisolone was initiated at $1 \mathrm{mg} / \mathrm{kg} /$ day, slowly tapered, and discontinued a

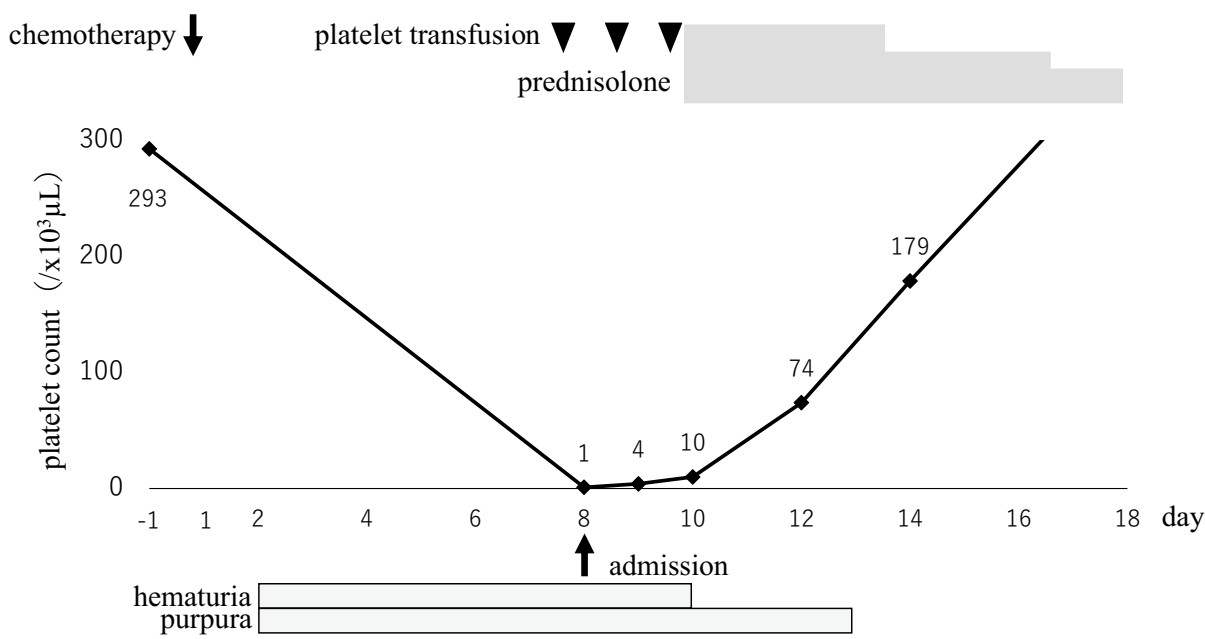

b

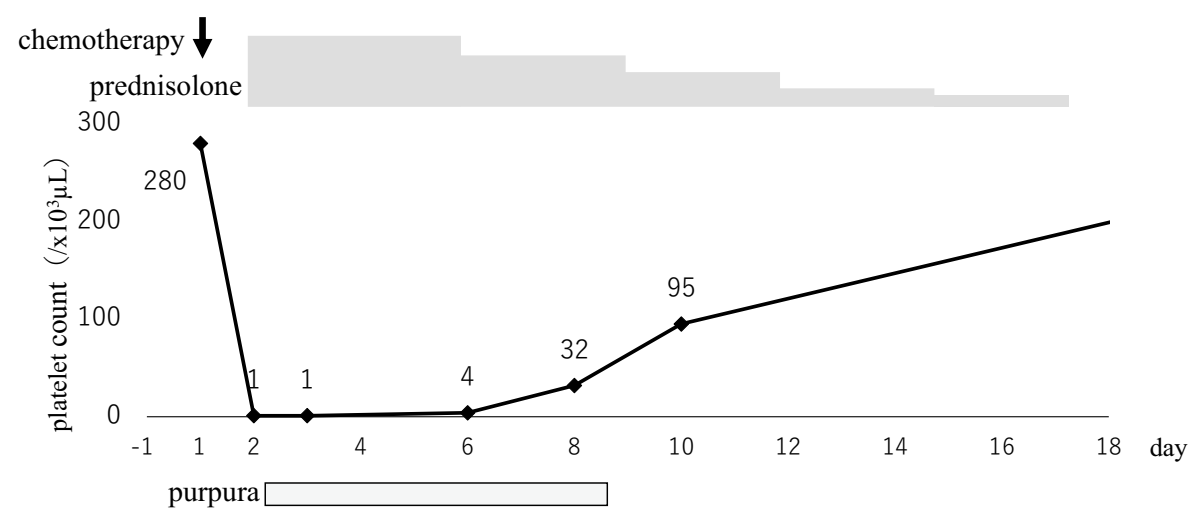


Table 1 List of published cases of drug-induced thrombocytopenia caused by trastuzumab

\begin{tabular}{|c|c|c|c|c|c|c|c|}
\hline Authors & Age & Sex & Cancer type & $\begin{array}{l}\text { Number of } \\
\text { cycles at } \\
\text { onset }\end{array}$ & Concurrent chemotherapy & $\begin{array}{l}\text { Platelet } \\
\text { nadir } \\
\left(/ \times 10^{3} \mu \mathrm{L}\right)\end{array}$ & Management \\
\hline Cathomas et al. [7, 9] & 54 & $\mathrm{~F}$ & MBC & 1 & None & 3 & IVIGs, steroids \\
\hline Parikh et al. [8] & 56 & $\mathrm{~F}$ & $\mathrm{EBC}$ & 1 & None & 2 & IVIGs \\
\hline Jara Sánchez et al. [10] & 37 & $\mathrm{~F}$ & MBC & 1 & Docetaxel, carboplatin & 3 & $\begin{array}{l}\text { PLT, IVIGs, ster- } \\
\text { oids, splenectomy }\end{array}$ \\
\hline Drudi et al. [11] & Not reported & $\mathrm{F}$ & $\mathrm{MBC}$ & 1 & Docetaxel & 7 & PLT, IVIGs, steroids \\
\hline Mantzourani et al. [12] & 56 & $\mathrm{~F}$ & EBC & 1 & None & 5 & IVIGs \\
\hline Aguirre et al. [13] & 63 & $\mathrm{~F}$ & $\mathrm{MBC}$ & Not reported & Paclitaxel & 22 & Steroids \\
\hline Pino et al. [14] & 70 & $\mathrm{~F}$ & MBC & 2 & Vinorelbine & 0 & PLT, IVIGs, steroids \\
\hline Zeng et al. [15] & 57 & $\mathrm{~F}$ & EBC & 21 & $\begin{array}{l}\text { Nab-paclitaxel, paclitaxel, car- } \\
\text { boplatin }\end{array}$ & 28 & Etamsylate, TPO \\
\hline Miarons et al. [16] & 70 & $\mathrm{~F}$ & EBC & 4 & Docetaxel & 39 & Steroids \\
\hline Luo et al. [17] & 39 & $\mathrm{~F}$ & $\mathrm{EBC}$ & 2 & Nab-paclitaxel & 3 & PLT, IL-11 \\
\hline Zhou et al. [18] & 35 & $\mathrm{~F}$ & $\mathrm{MBC}$ & 8 & Capecitabine & 1 & TPO, PLT, steroids \\
\hline Wang et al. [19] & 52 & $\mathrm{~F}$ & $\mathrm{EBC}$ & 1 & None & 1 & PLT, steroids \\
\hline Present case & 57 & $\mathrm{~F}$ & Gastric & 1 & Oxaliplatin, S-1 & 1 & PLT, steroids \\
\hline
\end{tabular}

$M B C$ metastatic breast cancer, $E B C$ early breast cancer, $P L T$ platelet transfusion, $I V I G$ intravenous immunoglobulin, $T P O$ thrombopoietin

numbers of polynuclear megakaryocytes in the bone marrow and an elevated level of platelet-associated $\operatorname{IgG}(\mathrm{PA}-\mathrm{IgG}$, $96 \mathrm{ng} / 10^{7}$ cells; normal range is less than $46 \mathrm{ng} / 10^{7}$ cells), the patient was diagnosed with drug-induced thrombocytopenia (DITP). After starting oral prednisolone $(1 \mathrm{mg} / \mathrm{kg})$ on day 10 , the platelet count recovered rapidly: $74,000 / \mu \mathrm{L}$ on day 12 and $179,000 / \mu \mathrm{L}$ on day 14 . Since we initially suspected oxaliplatin as a causal drug, S-1 was restarted as a monotherapy, followed by trastuzumab at a dose of $8 \mathrm{mg} /$ $\mathrm{kg}$ after a 3-week interval, without oxaliplatin. No infusion reaction was observed during or immediately after trastuzumab infusion, and the administration was completed safely. However, on the following day, purpura and petechiae reappeared throughout the patient's body, and hematologic tests revealed severe thrombocytopenia with a platelet count of $1000 / \mu \mathrm{L}$, which suggests that trastuzumab was responsible for the DITP (Fig. 2b). Since a significant tumor response to treatment was recognized on follow-up CT imaging (Fig. 1b), the patient continued S-1 and oxaliplatin treatment and no longer experienced severe thrombocytopenia.

\section{Discussion}

DITP is a rare adverse event associated with antibodymediated platelet destruction that is triggered by exposure to a causal drug. [1-3] Among several proposed mechanisms of DITP, it is highly probable that autoantibodies specific for glycoproteins (IIb/IIIa) on the platelet surface caused the destruction of platelets in the circulation of this patient. Besides the elevated levels of PA-IgG, [4] trastuzumab re-challenge immediately reproduced thrombocytopenia, which is consistent with the immune mechanism of DITP.

More than 300 drugs and agents, including oxaliplatin $[5,6]$, have been reported to cause DITP [1-3]. Thirteen cases of trastuzumab-related DITP have been reported thus far, including the present case (Table 1) [7-19]. The present case differs from other reported cases in that our patient had gastric cancer, and to our knowledge, this is the only case treated with the trastuzumab biosimilar CT-P6. According to the manufacturer (Nippon Kayaku, Tokyo, Japan), no incidents of DITP associated with this trastuzumab biosimilar have been reported other than our case. It is unknown whether biosimilar exhibit cross-reactivity to reference trastuzumab.

In conclusion, we report the first case of DITP associated with a trastuzumab biosimilar in a patient with gastric cancer, which provides useful insight into the practical management of DITP in molecular-targeted cancer treatment.

Acknowledgements The authors would like to thank Enago (www. enago.jp) for the English language review.

\section{Declarations}

Conflict of interest Yuichi Ando received a speaker honorarium from Chugai Pharmaceutical Co., Ltd. and research funding s from Chugai Pharmaceutical Co., Ltd., Yakult Honsha Co.,Ltd., Ono Pharmaceutical Co., Ltd., Novartis Pharma K.K., Bayer Holding Ltd., and Geo Holding Corporation. Other authors have no conflict of interest. 
Informed consent Written informed consent was obtained from the patient.

Open Access This article is licensed under a Creative Commons Attribution 4.0 International License, which permits use, sharing, adaptation, distribution and reproduction in any medium or format, as long as you give appropriate credit to the original author(s) and the source, provide a link to the Creative Commons licence, and indicate if changes were made. The images or other third party material in this article are included in the article's Creative Commons licence, unless indicated otherwise in a credit line to the material. If material is not included in the article's Creative Commons licence and your intended use is not permitted by statutory regulation or exceeds the permitted use, you will need to obtain permission directly from the copyright holder. To view a copy of this licence, visit http://creativecommons.org/licenses/by/4.0/.

\section{References}

1. Aster RH, Bougie DW (2007) Drug-induced immune thrombocytopenia. N Engl J Med 357(6):580-587

2. Bakchoul T, Marini I (2018) Drug-associated thrombocytopenia. Hematology Am Soc Hematol Educ Program 2018(1):576-583

3. Warkentin TE (2007) Drug-induced immune-mediated thrombocytopenia-from purpura to thrombosis. N Engl J Med 356(9):891-893

4. Kelton JG, Meltzer D, Moore J et al (1981) Drug-induced thrombocytopenia is associated with increased binding of $\mathrm{IgG}$ to platelets both in vivo and in vitro. Blood 58(3):524-529

5. Jardim DL, Rodrigues CA, Novis YAS, Rocha VG, Hoff PM (2012) Oxaliplatin-related thrombocytopenia. Ann Oncol 23(8):1937-1942

6. Pan E, Hsieh E, Piatek C (2018) Case Report: oxaliplatininduced immune-mediated thrombocytopenia. Case Rep Oncol 11(3):880-882

7. Cathomas R, Goldhirsch A, von Moos R (2007) Drug-induced immune thrombocytopenia. N Engl J Med 357(18):1870-1871

8. Parikh O, Neave F, Palmieri C (2008) Severe thrombocytopenia induced by a single infusion of trastuzumab. Clin Breast Cancer $8(3): 285-286$
9. Cathomas R, von Moos R (2009) Severe drug-induced thrombocytopenia after treatment with trastuzumab but not with lapatinib. Ann Oncol 20(9):1606-1607

10. Jara Sánchez C, Olier Gárate C, García-Donas Jiménez J, Peñalver PJ (2009) Drug-induced thrombocytopenia induced by trastuzumab: a special challenge in a curable disease. Ann Oncol 20(9):1607-1608

11. Drudi F, Gianni L, Fantini M, Ravaioli A (2010) Trastuzumabrelated thrombocytopenia: always a self-limiting complication? Ann Oncol 21(3):668-669

12. Mantzourani M, Gogas H, Katsandris A, Meletis J (2011) Severe thrombocytopenia related to trastuzumab infusion. Med Sci Monit 17(7):CS85-87

13. Aguirre E, Taberner T, Luaña A, Morales S, Llombart A (2013) Severe thrombocytopenia related to long-term trastuzumab exposure. Tumori 99(1):e1-2

14. Pino MS, Angiolini C, Fioretto L (2013) Severe thrombocytopenia after trastuzumab retreatment: a case report. BMC Res Notes 6:400

15. Zeng RC, Dai XX, Xie FY, Chen ED, Qu JM, Hu XQ (2014) Severe thrombocytopenia induced by second exposure to trastuzumab can be alleviated by prolonging the interval between treatments. Clin Breast Cancer 14(2):e69-e72

16. Miarons M, Velasco M, Campins L, Fernández S, Gurrera T, Lopez-Viaplana L (2016) Gradual thrombocytopenia induced by long-term trastuzumab exposure. J Clin Pharm Ther 41(5):563-565

17. Luo Q, Guo Z, Ye C (2019) Thrombocytopenia Induced by Herceptin. Am Surg 85(4):e222-e224

18. Zhou Q, Dong J, Jiang X, Pan Y, Han X (2020) Trastuzumabinduced thrombocytopenia after eight cycles of trastuzumab treatment. Open Med (Wars) 15(1):659-662

19. Wang X, Zhu X, Zou J, Zhang X, Kong X, Nie J (2021) Severe thrombocytopenia induced by trastuzumab rechallenge: a case report and literature review. J Clin Pharm Ther 46:1173-1177

Publisher's Note Springer Nature remains neutral with regard to jurisdictional claims in published maps and institutional affiliations. 